\title{
Comment on "Weyl fermions and the anomalous Hall effect in metallic ferromagnets"
}

\author{
David Vanderbilt \\ Department of Physics and Astronomy, Rutgers University, Piscataway, New Jersey 08854-8019, USA \\ Ivo Souza \\ Centro de Física de Materiales and DIPC, Universidad del País Vasco, 20018 San Sebastián, \\ and Ikerbasque Foundation, 48011 Bilbao, Spain \\ F. D. M. Haldane \\ Department of Physics, Princeton University, Princeton, New Jersey 08544, USA \\ (Received 14 December 2013; published 18 March 2014)
}

\begin{abstract}
We point out that, contrary to an assertion by Chen et al. [Phys. Rev. B 88, 125110 (2013)], the nonquantized part of the intrinsic anomalous Hall conductivity can indeed be expressed as a Fermi-surface property even when Weyl points are present in the band structure.
\end{abstract}

DOI: 10.1103/PhysRevB.89.117101

PACS number(s): 75.47.-m, 03.65.Vf, 71.90.+q, 73.43.-f

In a recent paper, Chen, Bergman, and Burkov (CBB) [1] challenged the claim that the nonquantized part of the intrinsic anomalous Hall conductivity (AHC) can be regarded as a Fermi-surface property [2]. In this Comment, we point out that CBB misrepresented the previous work and that the formal analysis of Ref. [2], as well as subsequent first-principles calculations based on Fermi-surface integrals [3], are in fact correct.

CBB start from their Eq. (4), an expression for the intrinsic $\mathrm{AHC}$ in terms of an integral of the Berry curvature over the occupied band manifold in the Brillouin zone (BZ). Following Ref. [3], they write this as

$$
\sigma_{x y}=\frac{1}{2 \pi} \int_{-\pi}^{\pi} d k_{z} \sigma_{x y}^{2 \mathrm{D}}\left(k_{z}\right),
$$

where $\sigma_{x y}^{2 \mathrm{D}}$ is the contribution arising from a slice of the BZ at a given $k_{z}$. They then point out that if $\sigma_{x y}^{2 \mathrm{D}}$ is evaluated as a sum of Berry phases computed as integrals over Fermi loops on the slice,

$$
\sigma_{x y}^{2 \mathrm{D}}\left(k_{z}\right)=\frac{e^{2}}{2 \pi h} \sum_{n} \oint d \mathbf{k} \cdot \mathbf{A}_{n \mathbf{k}}\left(k_{z}\right),
$$

where $\mathbf{A}$ is the Berry potential and the sum is over bands crossing the Fermi energy, then contributions from entirely filled bands can be missed. Particularly when isolated band crossings ("Weyl points") are present in the occupied manifold, they argue that Eq. (1) will then yield an incorrect result.

This is true as far as it goes. However, the Fermi-surface formulas proposed in Ref. [2] are not those of Eqs. (1) and (2) above. Instead, the formula proposed in Eq. (20) of Ref. [2] states that the nonquantized part of the AHC can be written, upon recasting the Hall conductivity as a vector, as

$$
\sigma=\frac{e^{2}}{(2 \pi)^{2} h} \sum_{\alpha} \int_{S_{\alpha}} d^{2} k[\mathcal{F}(\mathbf{k}) \cdot \hat{\mathbf{n}}(\mathbf{k})] \mathbf{k} .
$$

This takes the form of a sum of Fermi-surface integrals of the position $\mathbf{k}$ on the Fermi surface weighted by the surfacenormal component of the Berry curvature $\mathcal{F}=\boldsymbol{\nabla} \times \mathbf{A}$ of the band crossing the Fermi energy at k. (The above assumes that the Fermi sheets $S_{\alpha}$ do not touch the BZ boundary; the generalization to the case that they do is provided in Eq. (21) of Ref. [2].) CBB seem to have overlooked that this was the actual Fermi-surface expression proposed in Ref. [2]. The possible existence of Weyl points was carefully considered as part of the derivation of Eq. (3), which remains correct even when they are present.

There is also no reason for concern that published firstprinciples calculations of the AHC might be incorrect because of overlooking the subtleties discussed by CBB. Clearly, those that were based on volume integrals of the Berry curvature [4-6] are unaffected. (In this class, approaches based on gauge-invariant trace formulas [7] are particularly suited to the presence of Weyl points, since they remove the singularity entirely.)

Of more concern is the Fermi-loop calculation of Ref. [3], which was also based on Eqs. (1) and (2) above. Since Berry phases are only defined modulo $2 \pi$, those equations must be supplemented by a prescription for choosing the branch cuts as a function of $k_{z}$. CBB adopted a prescription in which the sum of Berry phases in Eq. (2) was equated with the two-dimensional (2D) integral of the Berry curvature over the occupied portions of the BZ for the partially filled bands only. This leads to unphysical step discontinuities in $\sigma_{x y}^{2 \mathrm{D}}$ at isolated $k_{z}$ values where a Weyl point between the last fully occupied and the first partially occupied band crosses the BZ slice, which CBB compensate for by adding a counterterm in their Eq. (8). Instead, in Ref. [3], the quantity $\sigma_{x y}^{2 \mathrm{D}}$ was chosen to be a continuous function of $k_{z}$. In this way, the extra nonquantized contributions from filled bands in Eq. (8) of CBB are automatically included, as illustrated below. In any case, the results of the Fermi-loop and Fermi-sea integration approaches were compared in Ref. [3] and found to agree.

As an instructive example, consider a nearly insulating crystal that is only metallic due to the presence of two small electron pockets arising from shallow Weyl points of opposite chirality located at $\mathbf{k}_{1}$ and $\mathbf{k}_{2}$ [8]. In this case, Eq. (8) of CBB includes a contribution to $\sigma_{x y}$ that is proportional to $\left(k_{2 z}-k_{1 z}\right)$, which would be missed in a naive implementation of Eqs. (1) and (2) above. This contribution is also included in 
Eq. (3) because the integral of $\mathcal{F} \cdot \hat{\mathbf{n}}$ over each Fermi-surface pocket is $\pm 2 \pi$, due to the enclosed Weyl points; in the limit of small pockets, the factor of $\mathbf{k}$ can be pulled out of the integral, providing the needed $\left(k_{2 z}-k_{1 z}\right)$ term. In the Fermi-loop approach of Ref. [3], one sets the branch choice of $\sigma_{x y}^{2 \mathrm{D}}$ arbitrarily at some reference $k_{z}$, and then insists on continuity as a function of $k_{z}$. In this example, one can set $\sigma_{x y}^{2 D}$ to zero for $k_{z}$ below both pockets; it will then rise continuously from 0 to $e^{2} / h$ while traversing the pocket around $\mathbf{k}_{1}$, then remain constant at $e^{2} / h$ until the second pocket is reached, where it will again return to zero. When averaged over all $k_{z}$, this will correctly give a contribution proportional to $\left(k_{2 z}-k_{1 z}\right)$.

In summary, we conclude that the nonquantized part of the intrinsic AHC is indeed correctly expressed as a Fermi-surface property in Eqs. (20) and (21) of Ref. [2], and that the methods used in previous calculations of the AHC are correct, even when Weyl points are present in the occupied band manifold.
[1] Y. Chen, D. L. Bergman, and A. A. Burkov, Phys. Rev. B 88, 125110 (2013).

[2] F. D. M. Haldane, Phys. Rev. Lett. 93, 206602 (2004).

[3] X. J. Wang, D. Vanderbilt, J. R. Yates, and I. Souza, Phys. Rev. B 76, 195109 (2007).

[4] Z. Fang, N. Nagaosa, K. Takahashi, A. Asamitsu, R. Matthieu, T. Ogasawara, H. Yamada, M. Kawasaki, Y. Tokura, and K. Terakura, Science 302, 92 (2003).
[5] Y. Yao, L. Kleinman, A. H. MacDonald, J. Sinova, T. Jungwirth, D. S. Wang, E. Wang, and Q. Niu, Phys. Rev. Lett. 92, 037204 (2004).

[6] X. J. Wang, J. R. Yates, I. Souza, and D. Vanderbilt, Phys. Rev. B 74, 195118 (2006).

[7] M. G. Lopez, D. Vanderbilt, T. Thonhauser, and I. Souza, Phys. Rev. B 85, 014435 (2012).

[8] K.-Y. Yang, Y.-M. Lu, and Y. Ran, Phys. Rev. B 84, 075129 (2011). 